\title{
Case series reporting the effectiveness of mycophenolate mofetil in treatment- resistant asthma
}

\author{
To the Editor:
}

Treatment-resistant asthma represents a significant unmet clinical need. Due to lack of therapeutic options many patients receive treatment with long-term oral steroids despite appreciation of the associated systemic adverse effects. While anti-IgE therapy with omalizumab affords benefit and there have been encouraging outcomes with monoclonal antibodies against interleukin (IL)-5 and IL-13 [1,2], all such treatments target T-helper cell type 2 (Th2) orientated disease in a sub-population of patients.

Mycophenolate mofetil (MMF) is a reversible inhibitor of inosine monophosphate dehydrogenase, an enzyme required by $\mathrm{T}$ - and $\mathrm{B}$-cells for guanine synthesis [3]. It is most commonly used to prevent transplanted organ rejection, but is increasingly prescribed to treat inflammatory disorders such as connective tissue disorders and systemic vasculitides.

In January 2009, a patient in our institution with lifelong severe treatment-resistant asthma, poorly controlled despite high-dose inhaled corticosteroids, regular oral steroids and nebulised bronchodilators, was prescribed MMF for co-existent undifferentiated vasculitis. Over the following year his disease improved dramatically with a reduction in hospital admissions, enabling a reduction in oral steroids with a concurrent decrease in weight, improvement in quality of life and exercise capacity. In view of this experience, another individual with severe asthma resistant to all standard therapies was placed on MMF. This patient also showed a dramatic clinical improvement. Subsequently we treated a further 20 patients with MMF with varying results to give a case series of 22, which we report here.

All patients started on MMF had been under specialist asthma care for at least 12 months and despite step 4 or 5 of asthma guidelines management had poor control, as measured by the asthma control questionnaire (ACQ), and frequent exacerbations (at least two per year) needing oral corticosteroids. In all patients, standard therapies were either not applicable or had failed; open label MMF was started while continuing their maintenance treatment. We did not restrict MMF therapy by atopic status, inflammatory biomarkers or smoking history; no patients were current smokers.

MMF, started at $500 \mathrm{mg}$ daily, was incremented by $500 \mathrm{mg}$ every 4 weeks. Doses over $500 \mathrm{mg}$ daily were given as divided doses. Dosing was to a maximum $3000 \mathrm{mg}$ daily, unless limited by adverse effects or stopped at a lower level due to a sufficiently favourable outcome. Patients were informed of potential risks, the experience in the department, and the "off-licence" use of the medication due to lack of trial data. Safety monitoring was undertaken at each dose level, examining full blood count, renal function and liver enzymes. MMF was stopped if total white cell count was $<3.5 \times 10^{9} \mathrm{~L}^{-1}$, neutrophils $<2 \times 10^{9} \mathrm{~L}^{-1}$, platelets $<150 \times 10^{9} \mathrm{~L}^{-1}$, serum creatinine $>140 \mu \mathrm{mol} \cdot \mathrm{L}^{-1}$ or serum alanine transferase rose to three times the upper limit of normal.

22 patients (nine males, mean age 50.6 years) were initiated on MMF. Six did not tolerate the drug early in treatment due to gastrointestinal side-effects, palpitations, dizziness or abnormal liver function tests. These patients failed to increment and stopped MMF (mean treatment duration 2 months, range 0.2-4 months). Long-term efficacy was not evaluable in these patients and stability of their disease without MMF could not be evaluated as they were commenced on other therapies or enrolled in clinical trials. Of the remaining 16 patients, all took MMF for at least 6 months, and are included in the analysis that follows, even if there was no objective or subjective benefit. 12 patients remain on MMF, four patients stopped the drug due to lack of efficacy (three patients no objective effect, one patient no subjective improvement). Median dose (range) of MMF achieved was 2000 (1000-3000) mg per day. Data, presented as before MMF and after MMF therapy, represents data collected when the decision to stop treatment was made, or up to September 1, 2012 in those who remained on therapy. Data is expressed as median (interquartile range) with paired data analysed using the Wilcoxon matched-pairs signed-rank test.

The patients generally had long-standing asthma, with median (interquartile range) duration of diagnosis of 25 (13.5-46) years, and had been under specialist asthma care for 36 (17-52) months. 14 achieved sputum 
induction prior to MMF. In these, the percentage of eosinophils was 4 (1.1-14.2)\% and neutrophils 37.3 (25.5-48.4)\%. Total serum IgE was $42.5(23.0-294.3) \mathrm{IU} \cdot \mathrm{mL}^{-1}, 13$ were skin prick test positive to common aeroallergens and $\mathrm{FeNO}$ at a flow rate of $50 \mathrm{~mL} \cdot \mathrm{min}^{-1}$ was $21.3(9.9-42.8) \mathrm{ppb}$. Duration of MMF therapy was 12.5 (7.3-19.0) months.

Following MMF therapy, forced expiratory volume in $1 \mathrm{~s}$ (FEV1) increased from 46.5 (34.3-72.3)\% pred to $70.1(46.0-76.0) \%$ pred $(\mathrm{p}=0.008)$, ACQ6 decreased from $3.4(2.5-4.1)$ points to $2.5(1.0-3.6)$ points $(\mathrm{p}=0.01)$ and the number of exacerbations in 6 months decreased from $4(3-6)$ to $1(0-3)(\mathrm{p}<0.001)$ (fig. 1). Peripheral blood eosinophil count decreased from $0.2(0.1-0.4) \times 10^{9} \mathrm{~L}^{-1}$ to $0.1(0.0-0.3) \times 10^{9} \mathrm{~L}^{-1}$ with MMF treatment $(p=0.05)$. There was no significant change in either oral $(10.0(0.0-18.8)$ to $5.0(0.0-13.8) \mathrm{mg}$ per day $(\mathrm{p}=0.38))$ or inhaled $(2800(1600-3850) \mu \mathrm{g}$ per day beclometasone dipropionate equivalent to
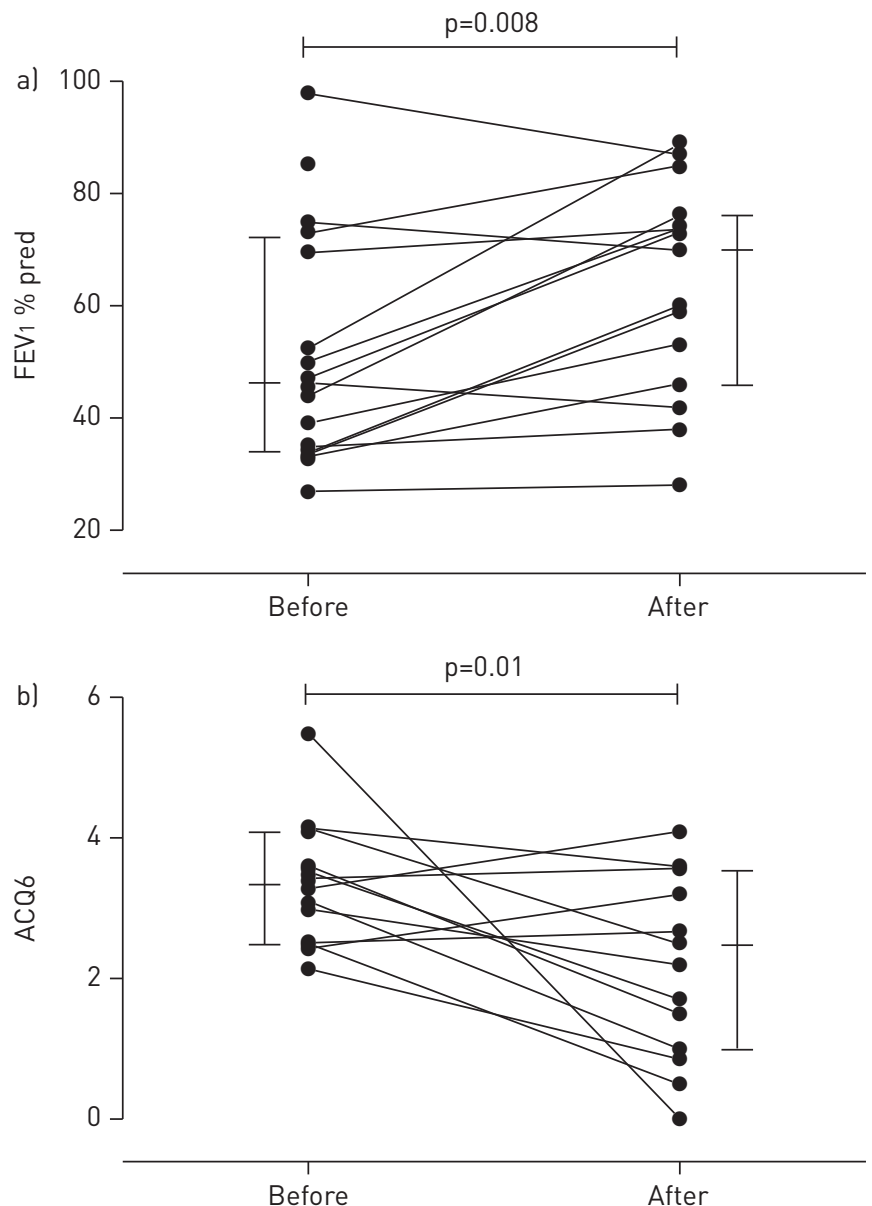

FIGURE 1 Change in forced expiratory volume in $1 \mathrm{~s}$ (FEV1), asthma control and exacerbation frequency following treatment with mycophenolate mofetil. Change in a) percentage predicted FEV1, b) quality of life (measured by asthma control questionnaire excluding spirometry (ACQ6)) and c) exacerbations requiring oral corticosteroid therapy in 6 months, before and after treatment with mycophenolate mofetil. p-values were calculated using Wilcoxon signed-rank test. Bars show medians and interquartile ranges.

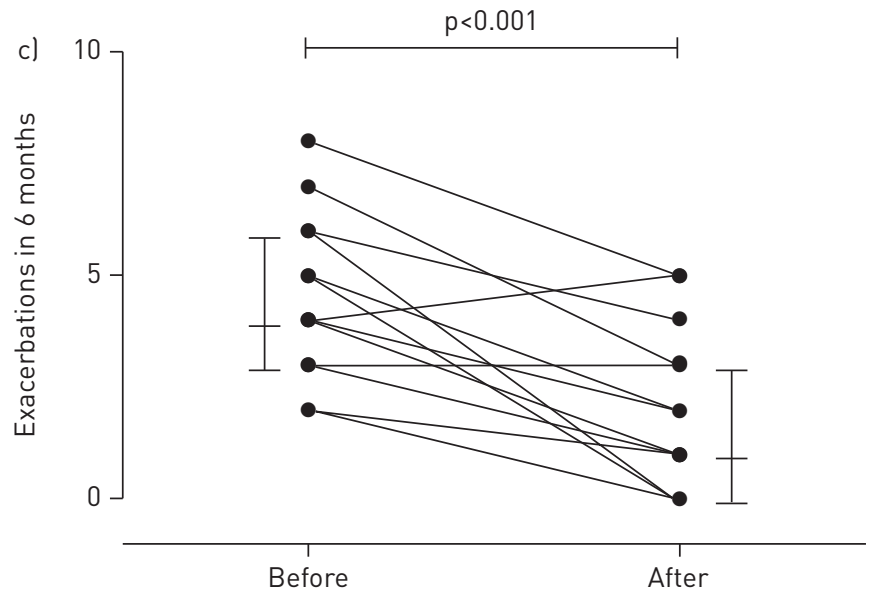


$1800(1600-2400) \mu \mathrm{g}$ per day $(\mathrm{p}=0.07))$ glucocorticosteroid doses. There was a small reduction in body mass index from $28.7(25.2-35.4) \mathrm{kg} \cdot \mathrm{m}^{-2}$ to $28.1(25.3-34.1) \mathrm{kg} \cdot \mathrm{m}^{-2}(\mathrm{p}=0.003)$. There were no significant correlations between any of the above outcomes and pre-treatment investigations listed above.

MMF improved many measures of asthma control, including ACQ6, FEV1 and exacerbation rates. These improvements were evident despite pre-existing therapy with high-dose inhaled glucocorticoids, longacting $\beta$-agonists and oral glucocorticoids in 11 of our patients. Overall, 12 out of the 22 patients that commenced MMF treatment tolerated and benefitted from this intervention. This represents the largest case series of MMF in treatment-resistant asthma. There has only been one previous single case report [4] and a report of beneficial experience in a Churg-Strauss-like syndrome [5].

MMF reduces IL-5 generation in animal models of asthma and also in peripheral blood mononuclear cells from asthmatics in vitro [6]. This alone, however, cannot account for the benefit in our patients as the antiIL-5 antibody, mepolizumab, does not improve ACQ or FEV1, despite decreasing asthma exacerbation frequency [1]. Broader T-cell targeting may be of greater benefit; consistent with this are reports of benefit with cyclosporine and azathioprine in treatment-resistant asthma [7].

It must be recognised, however, that these positive outcomes have arisen in an open label case series. Although most of these patients had been trialled with other novel therapies, including enrolment in formal trials, and MMF appears to have had an effect where these other medications had not, a placebo effect [8] cannot be excluded. Contextually it is also recognised that the findings from small studies are not always supported by larger formal trials, as with the soluble tumour necrosis factor- $\alpha$ receptor, etanercept, in severe asthma and that risk/benefit is an important consideration in the adoption of a new therapy [9].

Long-term safety of MMF prescribed alone is difficult to assess as it is commonly used in combination with calcineurin inhibitors and corticosteroids. Sole use of MMF is becoming more common, for example in lupus nephritis; in this context $\sim 25 \%$ of patients treated with MMF stop due to side-effects, while the incidence of upper respiratory tract infection is 35\%, arthralgia 25\% and leukopenia 23\% [10]. More concerning are potential haematological effects, which appear early in treatment and are reversible, as well as a small increased incidence of lymphoproliferative disease and nonmelanoma skin cancer. Our results should thus not be translated into a standard approach. Nevertheless the results to date with MMF are, in our view, sufficiently encouraging to support the conduct of a formal randomised placebo-controlled trial to further investigate this therapeutic approach as a new, potential, oral therapy for treatment-resistant severe asthma.

0 @ERSpublications

A case series using mycophenolate mofetil, a drug used in transplant medicine, suggests promise in treating severe asthma http://ow.ly/nuHTz

Christopher Grainge, Nivenka Jayasekera, Patrick Dennison, Hitasha Rupani, Ramesh Kurukulaaratchy and Peter Howarth

Dept of Respiratory Medicine, University Hospital Southampton, Southampton, UK.

Correspondence: P. Howarth, Clinical and Experimental Sciences, Mailpoint 810, South Academic Block, University Hospital Southampton, Tremona Road, Southampton, SO16 6YD UK. E-mail: P.H.Howarth@southampton.ac.uk

Received: Feb 112013 | Accepted: May 282013

Conflict of interest: Disclosures can be found alongside the online version of this article at www.erj.ersjournals.com

\section{References}

Pavord ID, Korn S, Howarth P, et al. Mepolizumab for severe eosinophilic asthma (DREAM): a multicentre, double-blind, placebo-controlled trial. Lancet 2012; 380: 651-659.

2 Corren J, Lemanske RF, Hanania NA, et al. Lebrikizumab treatment in adults with asthma. N Engl J Med 2011; 365 : 1088-1098.

3 Dall'Era M. Mycophenolate mofetil in the treatment of systemic lupus erythematosus. Curr Opin Rheumatol 2011; 23: 454-458.

4 Backer V, Hjardem E, Karlsmark T. Treatment with mycophenolat mofetil of steroid-dependent asthma-one case of severe asthma. J Allergy (Cairo) 2009; 2009: 821013.

5 Lau EMT, Cooper W, Bye PT, et al. Difficult asthma and Churg-Strauss-like syndrome: a cautionary tale. Respirology 2011; 16: 180-181.

6 Powell N, Till S, Bungre J, et al. The immunomodulatory drugs cyclosporin A, mycophenolate mofetil, and sirolimus (rapamycin) inhibit allergen-induced proliferation and IL-5 production by PBMCs from atopic asthmatic patients. J Allergy Clin Immunol 2001; 108: 915-917.

7 Redington AE, Hardinge FM, Madden J, et al. Cyclosporin A treatment and airways inflammation in corticosteroid-dependent asthma. Allergy 1998; 53: 94-98. 
9 Holgate ST, Noonan M, Chanez P, et al. Efficacy and safety of etanercept in moderate-to-severe asthma: a randomised, controlled trial. Eur Respir J 2011; 37: 1352-1359.

10 Dooley MA, Jayne D, Ginzler EM, et al. Mycophenolate versus azathioprine as maintenance therapy for lupus nephritis. N Engl J Med 2011; 365: 1886-1895.

\title{
Occupational asthma from sensitisation to 4,4-methylene-bismorpholine in clean metalworking fluid
}

\begin{abstract}
To the Editor:
European Respiratory Society guidelines on the management of work-related asthma recommend that occupational asthma with an allergic mechanism should be diagnosed by both identifying the workplace as the cause, and confirming sensitisation to the asthmagen by specific inhalational challenge (SIC), in the absence of any available specific IgE tests $[1,2]$. Used (contaminated) metalworking fluid is the usual cause of occupational asthma in exposed workers. We present the first case of occupational asthma due to the biocide additive 4,4-methylene-bismorpholine present in clean metalworking fluid.
\end{abstract}

A 54-year-old Kenyan male presented with a 2-year history of rhinitis, wheeze, dry cough and chest tightness, which were worse at night and while at work, and improved away from work on holiday. He had had rhinitis from grass pollen, perfumes and cleaning agents intermittently for 15 years, and acne due to metalworking fluid 5 years previously. There was no childhood or family history of asthma and he had never smoked cigarettes. He had been employed as a machine tool setter operator for 22 years manufacturing car axles. For the past 6 years he had worked on an enclosed computer numerical controlled milling, drilling and boring machine, with its own oil sump, using carbide-tipped tools. It was loaded by a robot but there was no delay between the end of machining and door opening, which produced a visible mist about $3 \mathrm{~m}$ from his work station. The machines used Fuchs Ecocool Ultralife A, a semi-synthetic metalworking fluid for aluminium alloys. Clinical examination and chest radiograph were unremarkable. Skin-prick allergy testing (SPT) revealed a $0 \mathrm{~mm}$ reaction to $0.9 \%$ saline and a $7 \mathrm{~mm}$ reaction to histamine hydrochloride $10 \mathrm{mg} \cdot \mathrm{mL}^{-1}$. There were positive reactions to grass pollen $(8 \mathrm{~mm}), \operatorname{dog} \operatorname{dander}(5 \mathrm{~mm})$, cladosporium $(4 \mathrm{~mm})$ and 4,4-methylene-bis-morpholine $(3 \mathrm{~mm})$, with borderline reactions to $5 \mathrm{mg} \cdot \mathrm{mL}^{-1}$ cobalt chloride $(2.5 \mathrm{~mm})$ and clean metalworking fluid $(2.5 \mathrm{~mm})$. Total IgE was $2048 \mathrm{kU} \cdot \mathrm{L}^{-1}$, white blood cell count $7.81 \times 10^{9} \mathrm{~L}^{-1}$ and eosinophil count was raised at $0.75 \times 10^{9} \mathrm{~L}^{-1}$. Two-hourly peak flow measurements made at home and work over 4 weeks were analysed using OASYS [3, 4]. The OASYS score was 3.4 and the area between the curves score was $23.4 \mathrm{~L} \cdot \mathrm{min}^{-1} \cdot \mathrm{h}^{-1}$ confirming a significant work effect (fig. 1a). Spirometry revealed a borderline obstructive ventilatory defect (forced expiratory volume in $1 \mathrm{~s}$ (FEV1) to forced vital capacity (FVC) ratio 70\%) with normal indices (FEV1 $2.75 \mathrm{~L}, 86 \%$ predicted; FVC $3.95 \mathrm{~L}, 100 \%$ predicted). While work-exposed, his fractional exhaled nitric oxide (FeNO) was raised at $71 \mathrm{ppb}[5,6]$ and nonspecific bronchial responsiveness (NSBR) to methacholine was normal ( $>4800 \mu \mathrm{g}$ by the Yan method [7]).

He underwent SIC after 3 weeks away from work. He was challenged with 7\% clean Ecocool Ultralife A metalworking fluid in water for a total of $50 \mathrm{~min}$ via a Turboneb II (Clement Clarke International Ltd, Harlow, UK) and Maxineb (Flexicare Medical Ltd, Mountain Ash, UK) nebuliser. Initial FEV1 was 2.63 L, which fell immediately by $20 \%$, recovering after $15 \mathrm{~min}$, and fell again at $3-11 \mathrm{~h}$ by $29.7 \%$, accompanied by asthmatic symptoms. Subsequent SIC to $0.7 \%$ 4,4-methylene-bismorpholine in water (Chemical Abstract Service (CAS) registry number 5625-90-1; occupational asthma hazard index 0.98) for a total of $50 \mathrm{~min}$ resulted in immediate sustained rhinitis symptoms, then a late fall in FEV1 of $16.5 \%$ from $2.36 \mathrm{~L}$, at 9-11 h after challenge (fig. 1b). He had a more than four-fold increase in NSBR to methacholine (pre-SIC cumulative dose that caused a $20 \%$ fall in FEV1 (PD20) $>4800 \mu \mathrm{g}$; 24-h post-SIC PD20 $587 \mu \mathrm{g}$ ), but no clinically significant change in FeNO (FeNO pre-SIC $77 \mathrm{ppb}$; post-SIC $70 \mathrm{ppb}$ ). He had negative SICs to the solvent 2,2-aminoethoxyethanol (CAS registry number 929-06-6; occupational asthma hazard index 0.41), $\mathrm{pH}$ stabiliser 2-di-butylaminoethanol (CAS registry number 102-81-8; occupational asthma hazard index 0.95 ) 